\title{
Chart for Determining the Effects of Ionospheric Tilts Using an Idealized Model $^{1}$
}

\author{
T. A. Croft and R. B. Fenwick \\ Contribution from Radioscience Laboratory, Stanford University, Stanford, Calif.
}

(Received June 15, 1963)

\begin{abstract}
In studying the tilted ionosphere, it is often useful to consider the action of an equivalent reflecting mirror as an approximate substitute for the more complex refracting layer of electrons. Although the analogy is crude, it permits relatively easy computation of results which provide insight into the action of the real ionosphere.

Using the mirror analogy, the effect of a tilted ionosphere on a radio wave has been calculated for a wide range of tilt angles and directions without approximations. The results are presented on eight charts, each of which is calculated for two particular values of the parameters which must be fixed prior to calculation (mirror height and the distance from transmitter to the point on the earth beneath the mirror). Examples of chart use and implications for long-range HF transmission are also discussed.
\end{abstract}

\section{Introduction}

Effective ionospheric tilts can be important in determining ray path geometry in $\mathrm{HF}$ propagation, particularly in situations where takeoff angles are small. A chart has been derived whereby the position of all rays originating from the ground may be determined in space after having been reflected from a tilted plane mirror. The charts relate the following four parameters to one another:

1. The tilt angle of the mirror.

2. The orientation of the mirror tilt azimuth with respect to the incident ray.

3. The point of intersection of the reflected ray with the earth (if the ray returns to earth).

4. The height above the earth, and the lateral displacement of the reflected ray perigee (if the ray does not return to earth).

Charts have been calculated for mirror heights which are representative of $F_{2}$-layer virtual reflection heights, and for transmitter-to-mirror distances which correspond to takeoff angles of only a few degrees.

These calculations were originally carried out to demonstrate the ease with which ionospheric tilts can create (or destroy) a "round-the-world" propagation mode in which rays travel between successive ionospheric reflections without returning to earth [Fenwick, 1963]. It is believed that the charts should be of use to the scientific community because they show the effects of ionospheric tilts which are neither purely longitudinal nor purely transverse.

\section{Geometry}

It is possible to gain insight into the role of effective ionospheric tilts in determining ray-path geometry by use of the simple geometric model

1 The research reported here was carried out under the Office of Naval Research contract Nonr 225(64) and ARPA Order 196-63. shown in figure 1. A ray from the transmitter is presumed to be reflected from a mirror at height $H$, with a tilt angle $\phi$ and tilt azimuth $\theta$, as shown. If the resultant ray reflected from this mirror intersects the earth's surface, the point of intersection has coordinates $L$ (the ground distance from the original oreat circle) and $D$ (the ground distance from the point on the earth's surface under the mirror reflection point). If the reflected ray misses the earth's surface, as shown in figure 2, a miss distance $M$ (i.e., perigee altitude) and path deviation $L$ are defined.

Given the distance from the transmitter to the point on the earth's surface under the mirror reflection point $D_{T}$, and given $H$, it is then possible to calculate $D, L$, and $M$ as functions of $\phi$ and $\theta$.

The results of eight such calculations are shown on figures 3 through 10 with selected values of $D_{T}$ and $H$ as given in table 1.

TABLE 1.

\begin{tabular}{l|l|l|l|l}
\hline \hline \multirow{2}{*}{ Mirror height, $H$} & \multicolumn{3}{|c}{ Mirror-transmitter distance, $D_{T}$} \\
\cline { 2 - 4 } & $1000 \mathrm{~km}$ & $1250 \mathrm{~km}$ & $1500 \mathrm{~km}$ & $1750 \mathrm{~km}$ \\
\hline $\mathrm{km}$ & & & & \\
\hline $350 \ldots 0$ & & & Fig. 5 & Fig. 8 \\
\hline 350 & Fig. 3 & Fig. 4 & Fig. 6 & Fig. 9 \\
\hline
\end{tabular}

\section{Examples of Chart Use}

Assuming that the maximum effective ionospheric tilt which one may expect to encounter normally is on the order of $2 \mathrm{deg}$, it is interesting to determine the effect of such a tilt on rays incident from several different aspect angles. Consider figure 6, where $H=300 \mathrm{~km}$ and the path length for a nontilted mirror reflection surface is $3000 \mathrm{~km}$ (i.e., $2 D_{T}$ ). 


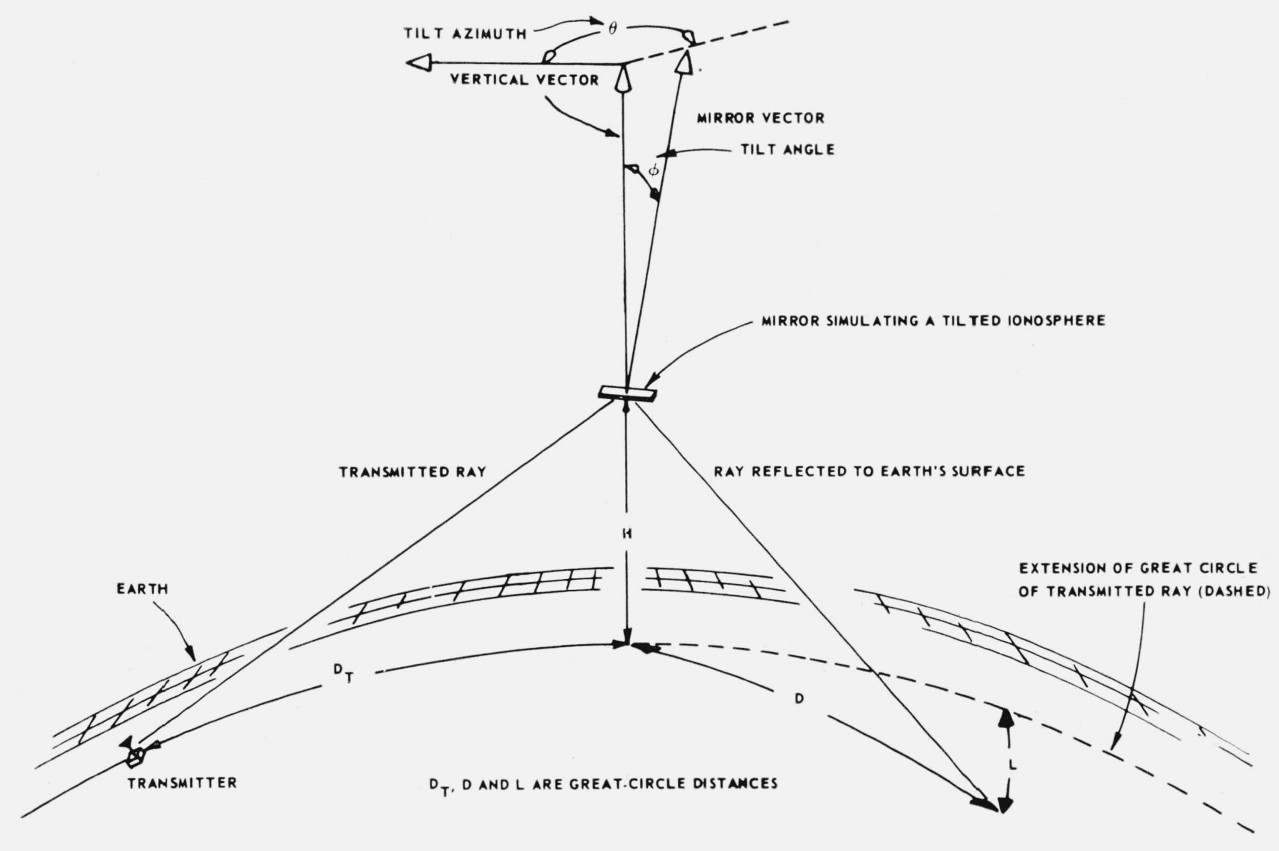

Figure 1. Geometry used to determine effects of ionospheric tilts on ray paths that return to earth.

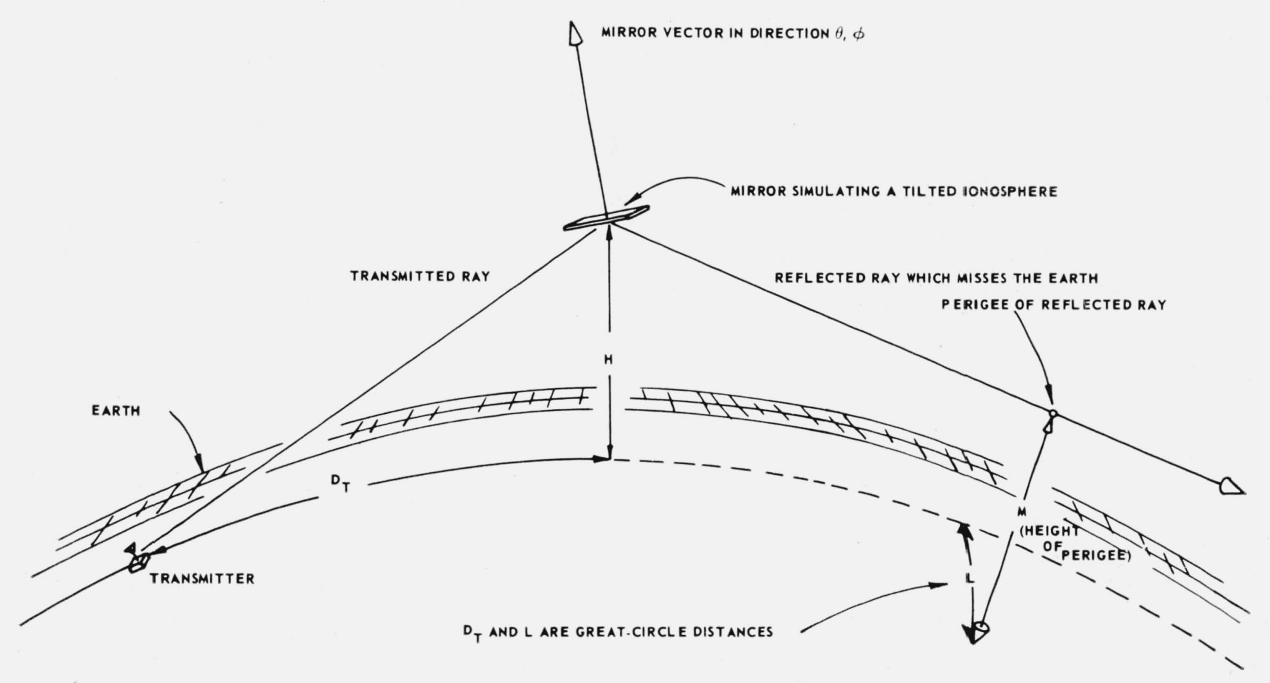

Figure 2. Geometry used for ray paths that miss the earth.

Assume $\phi=2 \mathrm{deg}$; then,

1. For $\theta=0^{\circ}$, a purely longitudinal "forward" tilt, the resultant ray path misses the earth $(M)$ by $109 \mathrm{~km}$ and is undeviated laterally $(L=0)$;

2. For $\theta=90^{\circ}$, a purely transverse tilt, $D=1517$ $\mathrm{km}$ and $L=33.5 \mathrm{~km}$. Hence the point of intersection of the ray with the earth is $33.5 \mathrm{~km}$ off the great-circle path defined by the transmitted ray.

3. For $\theta=180^{\circ}$, a longitudinal "backward" tilt, $D=922 \mathrm{~km}$ and $L=0$. The $2^{\circ}$ tilt reduced path length by about $570 \mathrm{~km}$.

Case I illustrates a situation in which an ionosphere-ionosphere reflection mode may be launched. It is possible, for instance, that such a mode would not return to the earth's surface until meeting a comparable tilt with $\theta=180^{\circ}$. 


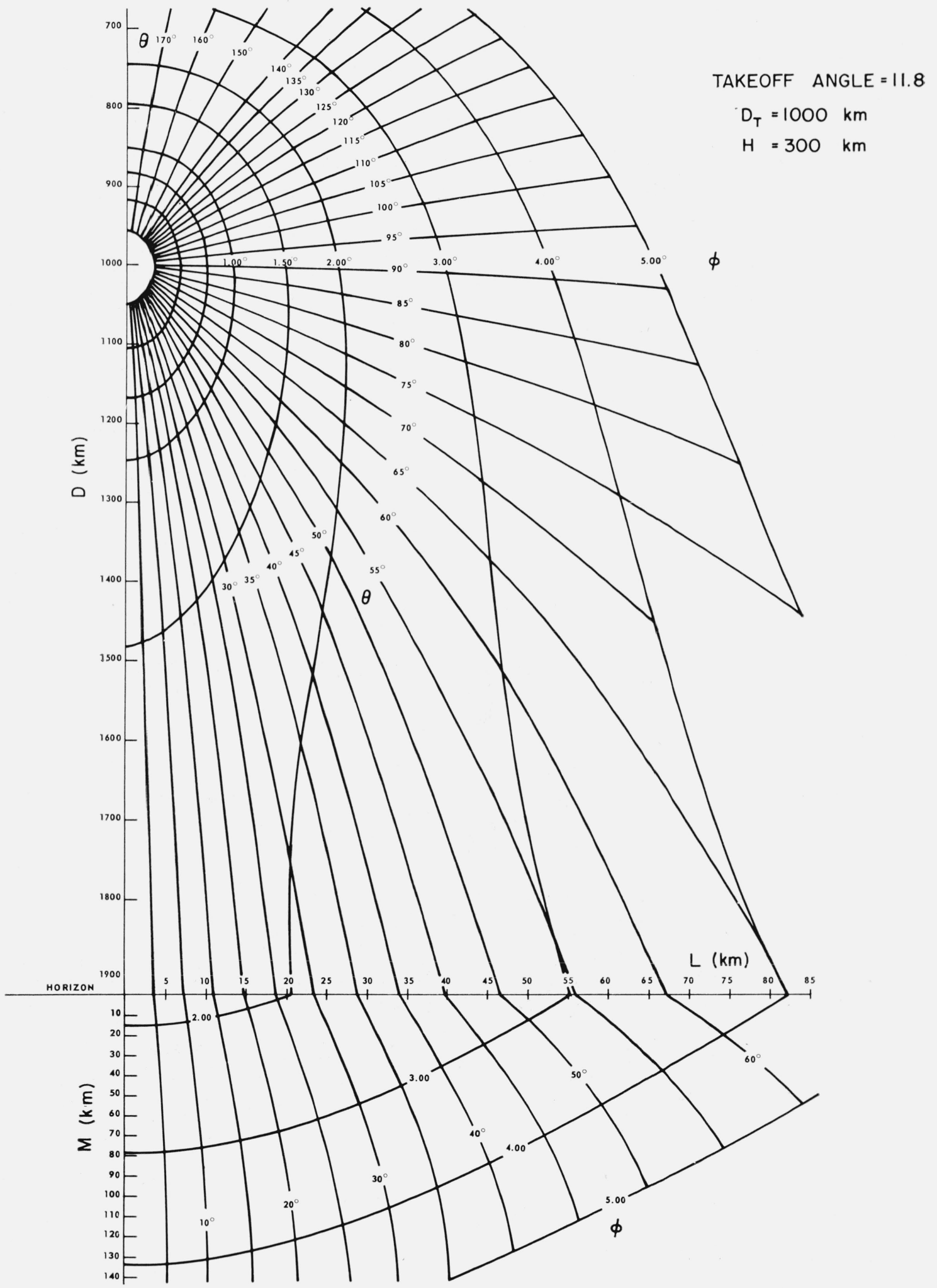

Figure 3. Computed effects of ionospheric tilts from the idealized model for $\mathrm{H}=300 \mathrm{~km}, \mathrm{D}_{\mathrm{T}}=1000 \mathrm{~km}$. 


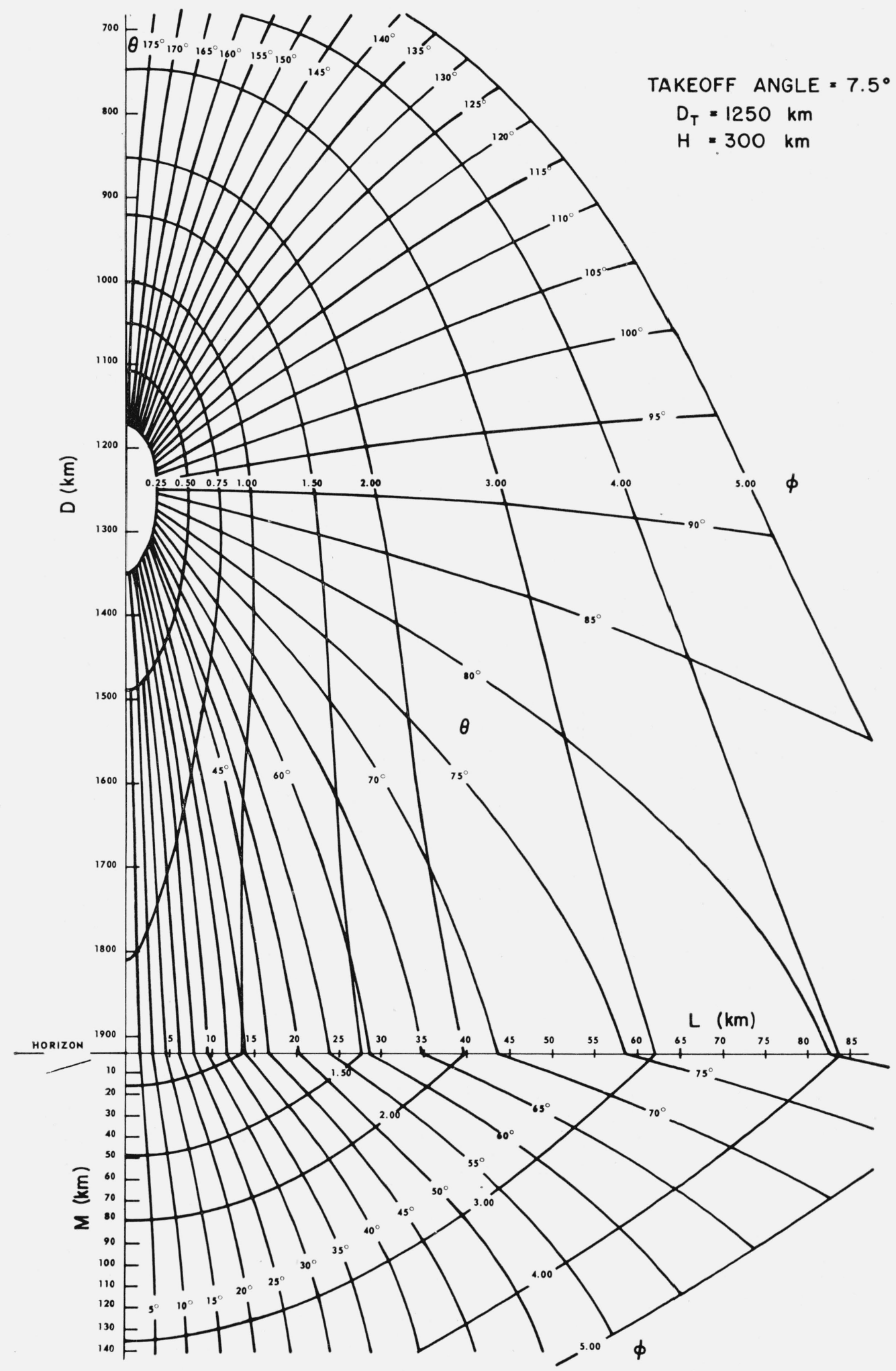

Figure 4. Computed effects of ionospheric tilts from the idealized model for $\mathrm{H}=300 \mathrm{~km}, \mathrm{D}_{\mathrm{T}}=1250 \mathrm{~km}$. 


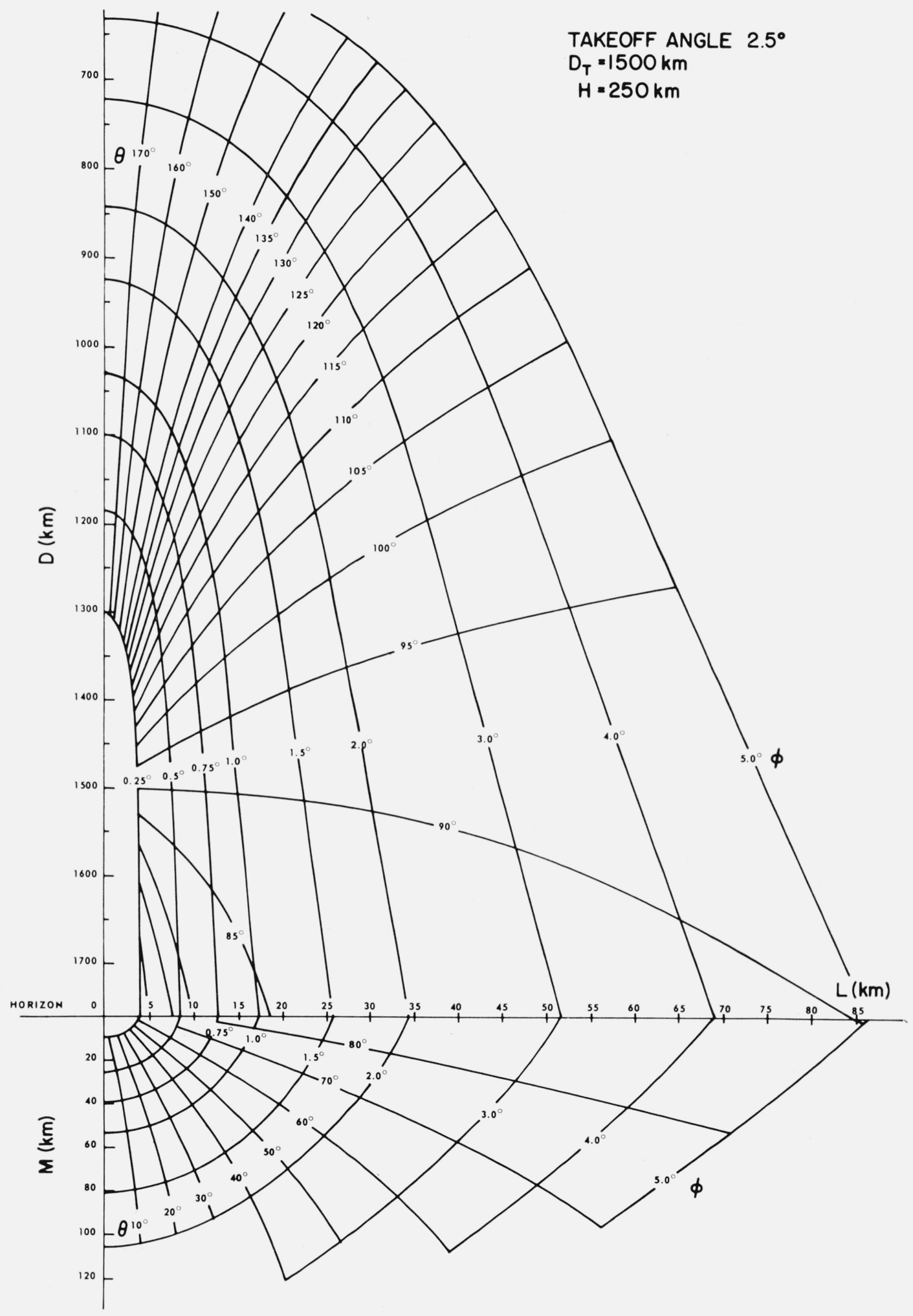

Figure 5. Computed effects of ionospheric tilts from the idealized model for $\mathrm{H}=250 \mathrm{~km}, \mathrm{D}_{\mathrm{T}}=1500 \mathrm{~km}$. 


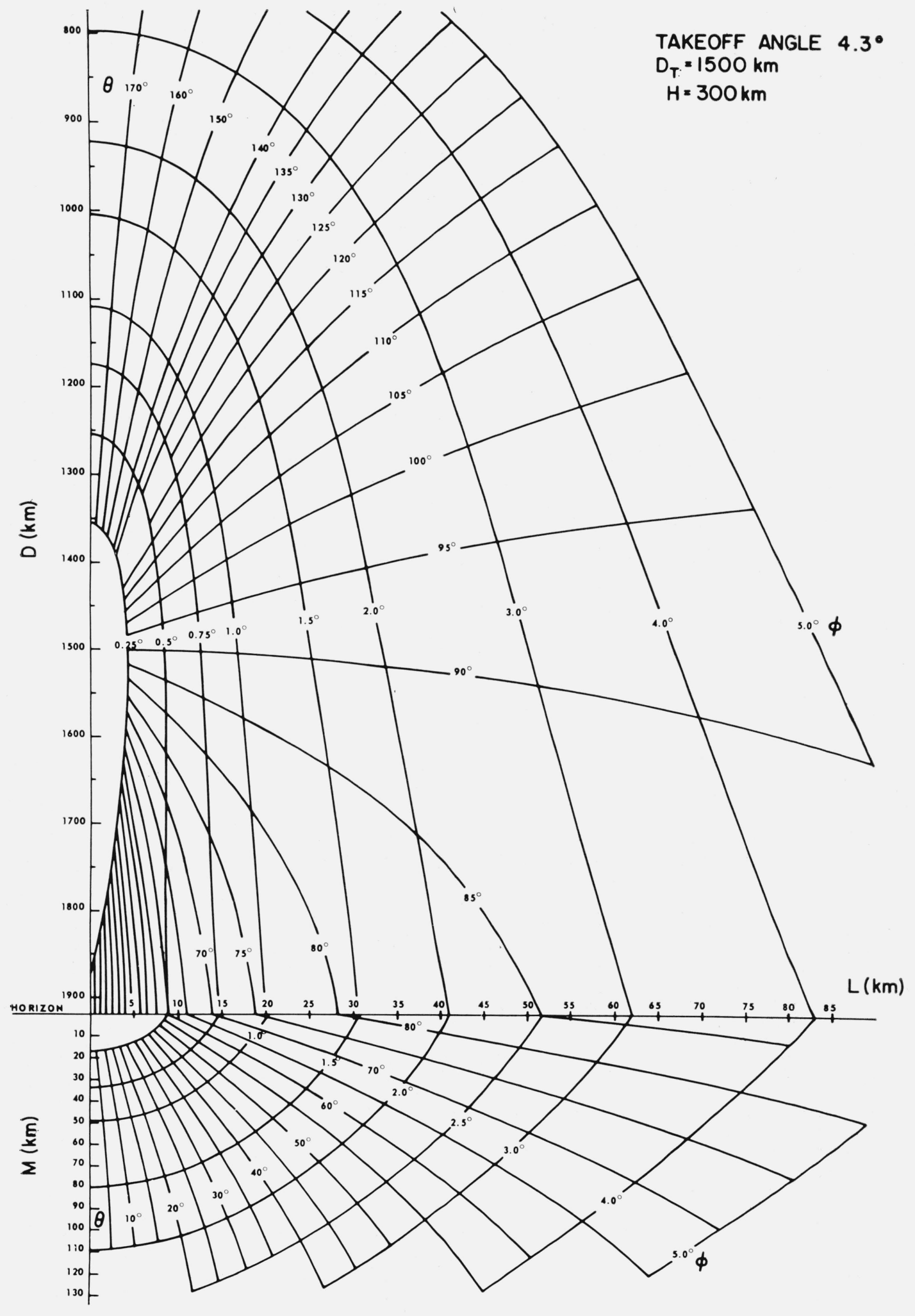

Figure 6. Computed effects of ionospheric tilts from the idealized model for $\mathrm{H}=300 \mathrm{~km}, \mathrm{D}_{\mathrm{T}}=1500 \mathrm{~km}$. 


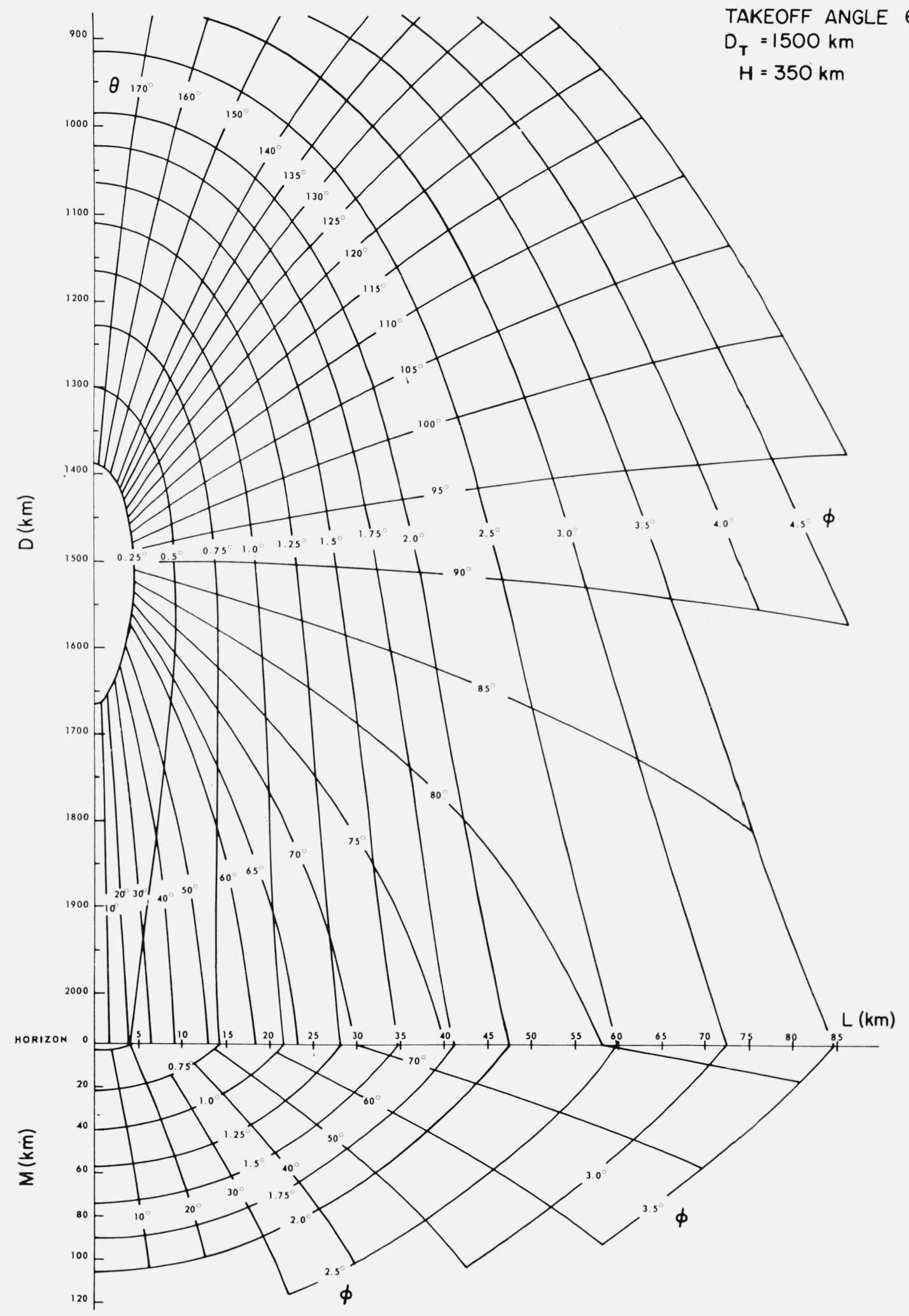

Figure 7. Computed effects of ionospheric tilts from the idealized model for $\mathrm{H}=350 \mathrm{~km}, \mathrm{D}_{\mathrm{T}}=1500 \mathrm{~km}$. 


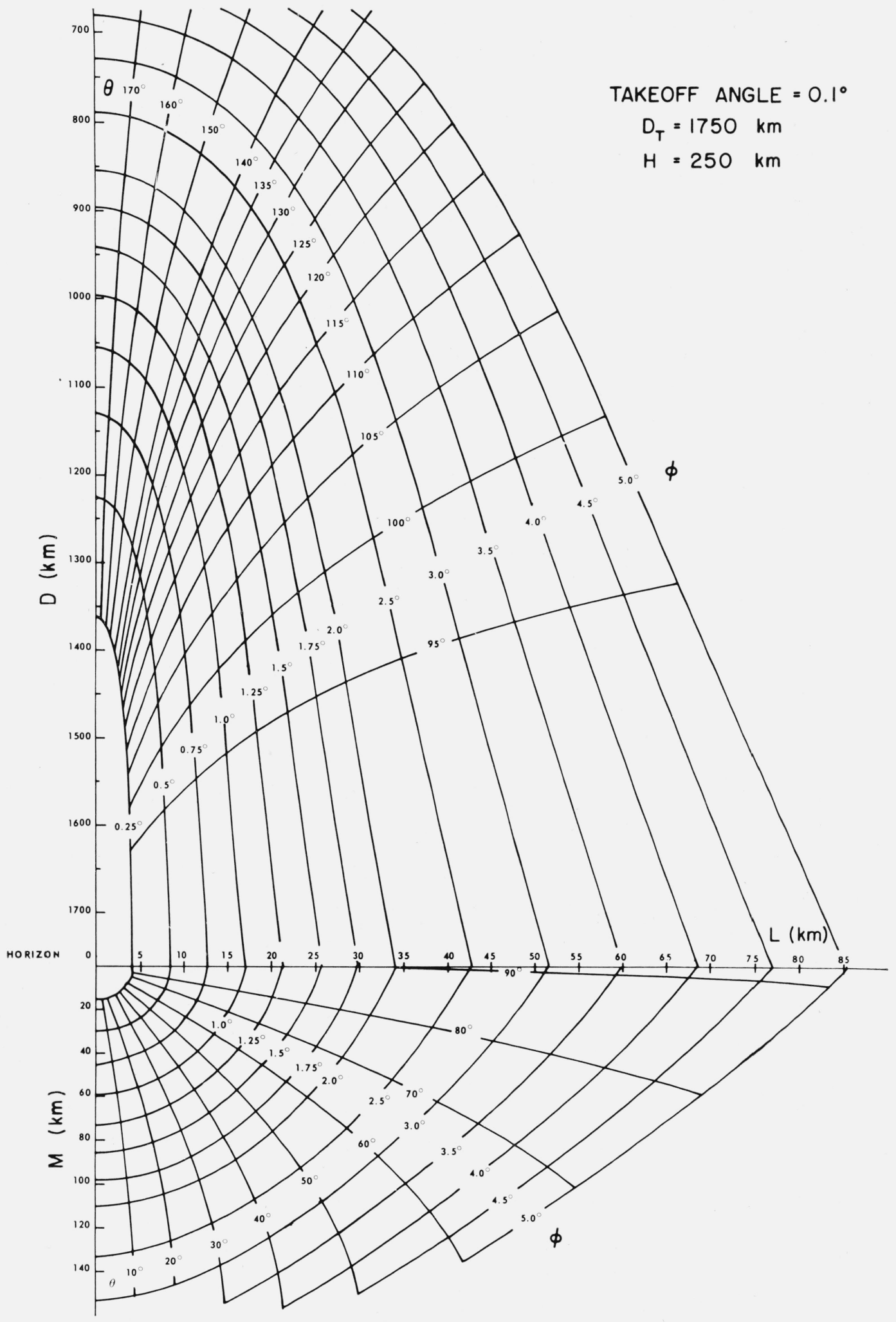

Figure 8. Computed effects of ionospheric tilts from the idealized model for $\mathrm{H}=250 \mathrm{~km}, \mathrm{D}_{\mathrm{T}}=1750 \mathrm{~km}$. 


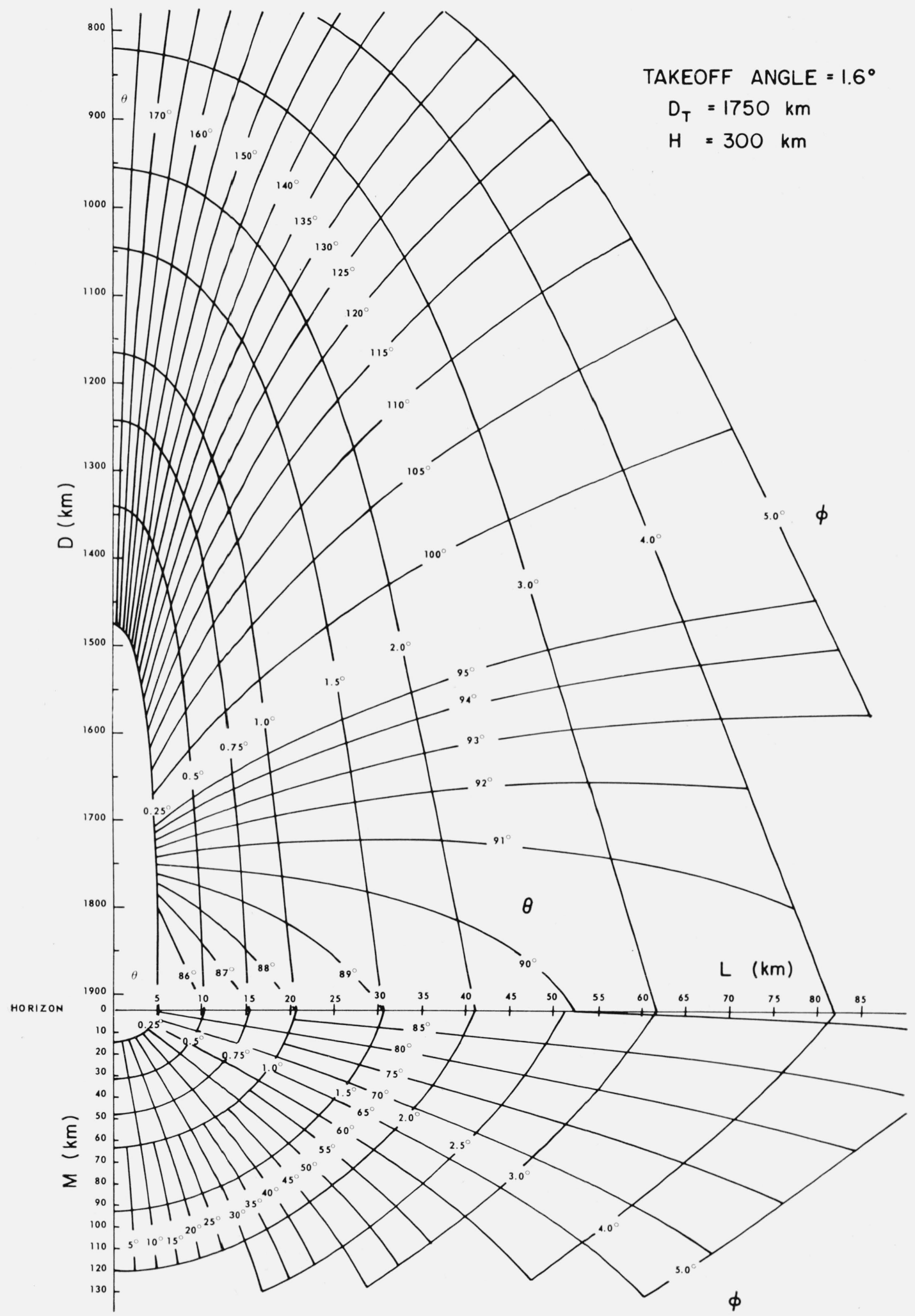

Figure 9. Computed effects of ionospheric tilts from the idealized model for $\mathrm{H}=300 \mathrm{~km}, \mathrm{D}_{\mathrm{T}}=1750$. 


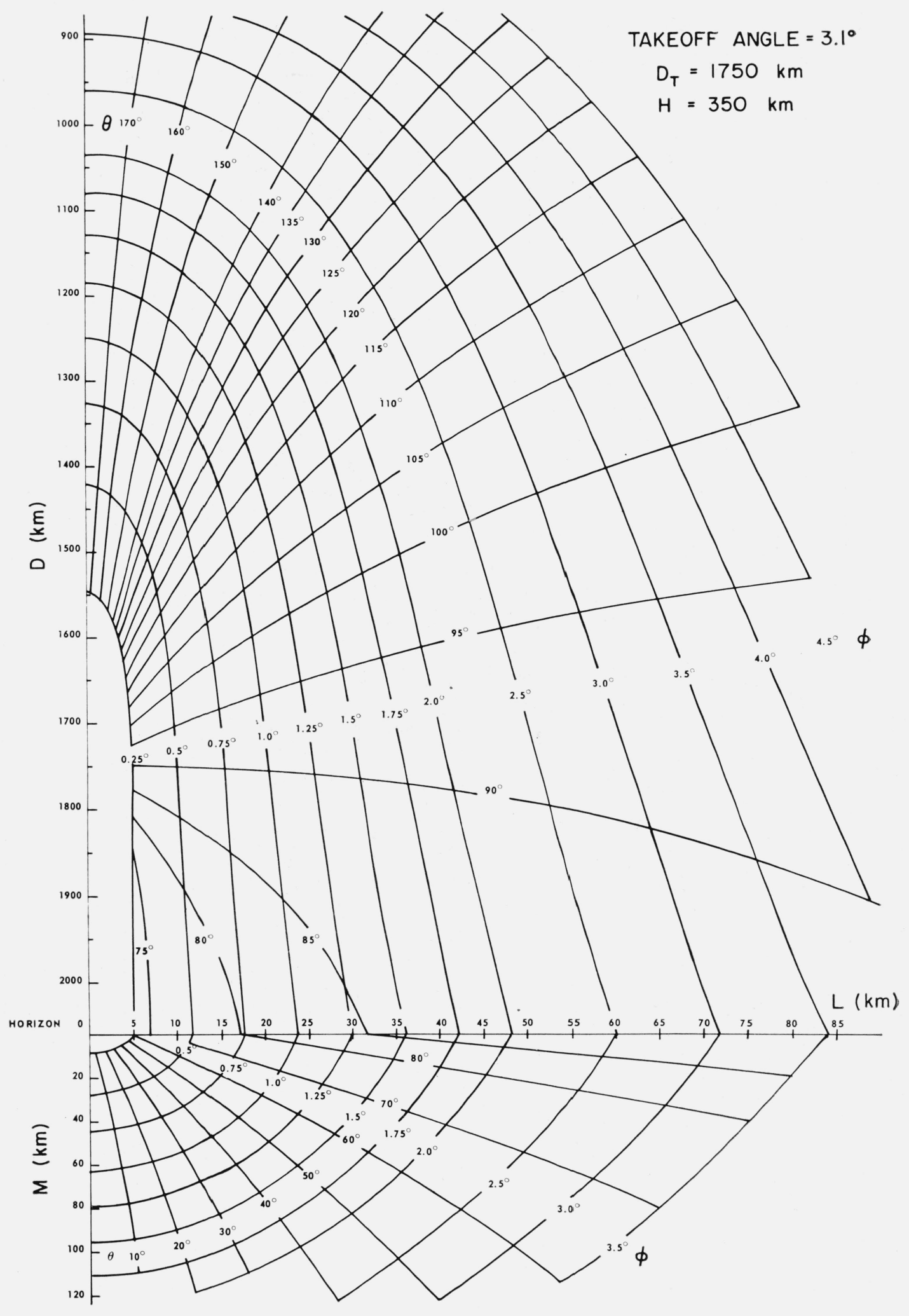

Figure 10. Computed effects of ionospheric tilts from the idealized model for $\mathrm{H}=350 \mathrm{~km}, \mathrm{D}_{\mathrm{T}}=1750$. 
Notice in particular that the $2^{\circ}$ tilt will cause a ray to miss the earth for all tilt azimuths between $0^{\circ}$ and $82.5^{\circ}$. Since the charts show only half of a figure which would have mirror symmetry about the vertical, it follows that all tilt azimuths between $+82.5^{\circ}$ and $-82.5^{\circ}$ can create an ionosphere-ionosphere reflection mode. This represents 46 percent of the entire $360^{\circ}$ range of azimuths.

Case II illustrates a situation in which a ray is deviated laterally, causing off-great-circle propagation to take place.

It can also be seen, for example, that 13 percent of the azimuth range of a $2^{\circ}$ tilt will cause rays to return to earth with a lateral deviation of at least $20 \mathrm{~km}$. Because of the chart symmetry, another 13 percent will cause $20 \mathrm{~km}$ or more deviation but in the opposite direction.

Case III illustrates a situation in which greatly unequal hop lengths occur in a one-hop geometry. Such a situation is likely to occur, for instance, when rays enter the daylight hemisphere from the night hemisphere transverse to the twilight line.

Here it is seen that a $2^{\circ}$ tilt reduces $D$ to $1000 \mathrm{~km}$ or less for azimuths between plus and minus $140^{\circ}$, or 22 percent of the azimuth range. For a $3^{\circ}$ tilt, 32 percent of the azimuth range will cause this degree of path length reduction.

Thus one could derive from the charts conclusions of the following nature: For $H=300 \mathrm{~km}, D_{T}=1500$ $\mathrm{km}, \phi$ (tilt) $=2^{\circ}$, and random tilt azimuth

1. There is a 0.46 probability that the reflected ray will miss the earth
2. There is a 0.26 probability that $L$ will exceed $20 \mathrm{~km}$

3. There is a 0.22 probability that the ray will return to earth within $1000 \mathrm{~km}$ of the mirror (as measured along a great circle).

\section{Conclusion}

A chart has been derived which permits estimation of the effects on the paths of $\mathrm{HF}$ radio waves when ionospheric tilts are encountered. The authors are not aware of any other comprehensive description of the action of tilts in other that the simplified cases of purely longitudinal or purely lateral tilt azimuth. It is assumed that the reflection takes place at a tilted plane mirror, but otherwise no approximations are involved. Details of the derivation, plus the computer program used to produce the charts, have been given by Fenwick [1963]. Interested workers could easily modify this program if they desire to study some other parameter, such as the angle of incidence of the downcoming ray.

\section{Reference}

Fenwick, R. B. (Apr. 1963), Round-the-world high-frequency propagation, TR 71, Radioscience Laboratory, Stanford University, Contract Nonr $225(64)$.

(Paper 67D6-302) 